Europhys. Lett., 60 (2), pp. 174-180 (2002)

\title{
Transport of modulated phases by pumping
}

\author{
L. M. Floría ${ }^{1,2}$, F. FAlo ${ }^{1,3}$, P. J. Martínez ${ }^{1,4}$ and J. J. Mazo ${ }^{1,3}$ \\ 1 Department of Theory and Simulation of Complex Systems, ICMA \\ CSIC-Universidad de Zaragoza - E-50009 Zaragoza, Spain \\ 2 Mathematics Institute, University of Warwick - Coventry CV4 7AL, UK \\ 3 Departamento de Física de la Materia Condensada, Universidad de Zaragoza \\ E-50009 Zaragoza, Spain \\ 4 Departamento de Física Aplicada, Universidad de Zaragoza \\ E-50009 Zaragoza, Spain
}

(received 16 May 2002; accepted in final form 30 July 2002)

PACS. 05.45.-a - Nonlinear dynamics and nonlinear dynamical systems.

PACS. 05.60.-k - Transport processes.

PACS. 05.45.Yv - Solitons.

\begin{abstract}
We give here some definite answers to the question on the possibility of transport of modulated phases by homogeneous pumping of (pinning) energy. They are presented for the convex class of the Frenkel-Kontorova model (discrete sine-Gordon equation). Our approach leads to a rigorous characterization of the transport under dissipative evolution and large pumping time scales. We also show an explicit example of computed phase diagram of the flow. When combined with Discommensuration Theory, our formal approach (essentially rooted in Aubry-Mather theory) gives an exact account of the features and singularities of this complex phase diagram in generic physical terms.
\end{abstract}

Modulated phases in physical systems arise from the competition between different interactions contributing to the system energy, each one imposing its own length scale [1-3]. For discrete modulated structures, it is often the case that they are localized (pinned) due to the existence of Peierls-energy barriers which prevent the modulated phase to slide. The height of the Peierls barrier depends on the relative strength $K$ of the competing interactions, that can often be externally controlled (by, e.g., monitoring an external field, or pressure, ...).

We are concerned here with the possibility of transport of modulated phases by (deterministic) cyclic variation of the relative strength $K(t)$ of the competing interactions: Is there any stationary flow of the distribution density of the modulated variable? In the affirmative, how does the flow depend on physical parameters? What specific symmetries (or lack of them) of the modulating field should be required? What kind of role discommensurations (kinks, solitons) play in the transport?

In order to give some definite and physically meaningful answers to these questions, we address them for the convex class of the Frenkel-Kontorova model $[4,5]$. Our choice is justified 

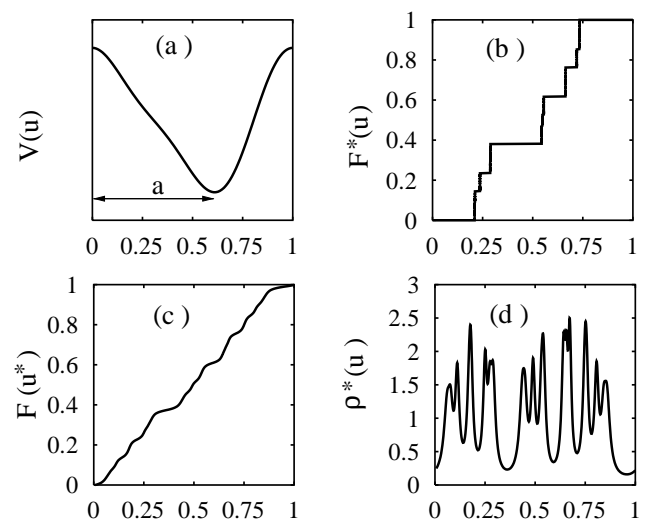

Fig. 1 - Graphs of: (a) The potential function $V(u)=(2 \pi)^{-2}[\sin (2 \pi u)+0.22 \sin (4 \pi u)]$, used in the numerical computations shown in the rest of figures. We have shifted the origin to have the maximum in 0. (b) Distribution function modulo $1, F^{*}(u)$, of the equilibrium modulated phase at $K=2$ for golden irrational $\omega=(\sqrt{5}-1) / 2$. (c) Equilibrium d.f. $(\bmod 1)$ for golden irrational at $K=0.6$. (d) Distribution density $\rho^{*}(u)=\frac{\mathrm{d} F^{*}}{\mathrm{~d} u}$ corresponding to (c).

by the prominent role that this model plays in our current physical understanding of modulated phases in Condensed Matter Theory $[1,6]$, and its central interest (discrete sine-Gordon equation) in Nonlinear Physics [7].

In the Frenkel-Kontorova (FK) model a modulated real variable $u_{n}$ is defined at each site $n$ of a one-dimensional macroscopic lattice. One can visualize $u_{n}$ as the position of a nonlinear oscillator. The two competing interactions are the pinning potential $V(u)$ (a periodic function of period 1) and the nearest-neighbour interaction energy $W(\Delta u)$, which will be assumed to be a convex function of interspacing $\Delta u$. Formally, the system energy of the FK model is

$$
H=\sum_{n}\left[K V\left(u_{n}\right)+W\left(u_{n+1}-u_{n}\right)\right]
$$

where the parameter $K$ controls the relative strength of the competing interactions: $V$ favouring integer values of $\Delta u$, and $W$ favouring a constant value $\omega$ of $\Delta u$, fixed by boundary conditions. We choose to work at fixed (arbitrary) modulating wave vector $\omega=\left\langle u_{n+1}-u_{n}\right\rangle=$ $\lim _{N \rightarrow \infty} N^{-1} \sum_{n}\left(u_{n+1}-u_{n}\right)$. Commensurate phases correspond to rational values of $\omega=p / q$, while incommensurate phases have irrational modulating wave vector.

For us, $K(t)$ will be a periodic function of time $(\operatorname{period} T)$ and we will consider dissipative evolution (dynamics) of the modulated structures $\left(u_{n}\right)$ :

$$
\dot{u}_{n}=-\frac{\partial H}{\partial u_{n}} .
$$

It turns out $[8,9]$ that under these circumstances (eqs. (1) and (2) with $W$ convex) the analysis of the dynamics of configurations $\left(u_{n}\right)$ can be conveniently reduced to an attracting invariant subset of configurations $S_{\mathrm{RO}}$ that is characterized by certain cross-avoiding property: No configuration $\left(u_{n}\right)$ in $S_{\mathrm{RO}}$ crosses any of its own translates $\left(u_{n}^{\prime}\right)=\left(u_{n+r}-s\right)(r, s$ arbitrary integers). Non crossing means that there are not $n \neq m$ with $u_{n}^{\prime}\left\langle u_{n}\right.$ and $\left.u_{m}^{\prime}\right\rangle u_{m}$. Configurations with this property are technically known as rotationally ordered (RO).

A convenient formal tool in the description of a configuration $\left(u_{n}\right)$ is the distribution function $F(u)$ of its fractional parts $\operatorname{Frac}\left(u_{n}\right)$, called distribution function modulo 1 of the 
configuration $[10,11]$, abbreviated d.f. $(\bmod 1) . F(u)$ is a nondecreasing and left continuous function, with $F(0)=0$ and $F(1)=1$. In fig. 1 we show examples of d.f. $(\bmod 1)$ of some equilibrium configurations. It is convenient to think of $F(u)$, defined originally on the interval $[0,1]$, as extended to the entire real axis by the formula $F(1+u)=1+F(u)$.

Any nondecreasing and left continuous function $F(u)$ with $F(0)=0$ and $F(1)=1$ can be associated to a one-parameter family of cross-avoiding configurations that do not cross each other, by the following construction:

$$
\left(u_{n}\right)^{\alpha}=F^{-1}(n \omega+\alpha) \text { for all } n,
$$

where $F^{-1}$ (the inverse function of the extended $F$ ), is usually termed hull (or modulating) function [5] of the family and $\alpha$ is an arbitrary phase.

Our goal is to obtain precise characterizations of the stationary flow,

$$
\mathcal{J}=T^{-1} \int_{0}^{T}\left\langle\dot{u}_{n}\right\rangle \mathrm{d} t
$$

which is formally related to a global rotation of the d.f. during the cycle. In order to obtain explicit expressions for the flow $\mathcal{J}$ we restrict consideration to analytical potential functions $V(u)$ possessing a single minimum per period located at $u=a(\bmod 1)$, and maxima at integer values of $u$. Regarding $K(t)$, we take it to be a squared-wave of period $T=\tau_{\text {on }}+\tau_{\text {off }}$ which takes alternatively the values $K$ and 0 . When both $\tau_{\text {on }}$ and $\tau_{\text {off }}$ are large compared to the characteristic relaxation times to equilibrium and $\omega$ is irrational, the d.f. evolves cyclically between $F(u)=u$ (equispaced configurations, when off-equilibrium is reached) and the equilibrium d.f. $F^{*}(u)$ corresponding to $K V(u)$ and commensurability $\omega$.

To compute the flow, think of an observer measuring the density of sites (or oscillators) for which $u_{n}(\bmod 1)$ pass through the value $u=0$ during both semicycles, $\mathcal{J}_{\text {off }}^{0}$ and $\mathcal{J}_{\text {on }}^{0}$. The sum of both partial local flows is the flow $\mathcal{J}(K, \omega)$.

$\mathcal{J}_{\text {off }}^{0}$ is essentially determined by the conservation of the distribution centroid $u_{\mathrm{cm}}(K, \omega)=$ $\int_{0}^{1} u \mathrm{~d} F_{K, \omega}^{*}$, where $F_{K, \omega}^{*}$ is the d.f. for the values $(K, \omega)$ of the model parameters. Elementary number theory (i.e. arithmetic) methods over this consequence of the Newtonian character of the nearest-neighbour interaction $W(\Delta u)$, leads to the exact expression for rational structures $\omega=p / q$,

$$
\mathcal{J}_{\text {off }}^{0}=q^{-1} \operatorname{Int}\left[q\left(u_{\mathrm{cm}}-\frac{1}{2}\left(1-\frac{1}{q}\right)\right)\right]
$$

leading to the following results for the incommensurate local flow as a function of $\omega$ :

$$
\mathcal{J}_{\text {off }}^{0}(\omega)=u_{\mathrm{cm}}-\frac{1}{2}
$$

where $u_{\mathrm{cm}}(\omega)$ is generally a continuous function of $\omega$ (from the Aubry-Mather theory see, e.g., $[12])$.

For the on-semicycle the local flow $\mathcal{J}_{\text {on }}^{0}$ is connected not only to $u_{\mathrm{cm}}$ but also to a quantity $\Delta$ which is related to the intersection of the "equispaced configuration" manifold (equilibrium initial conditions before on-semicycle) with the stable manifold of the saddle configuration lying on the top of the Peierls barrier between two contiguous (commensurate) equilibria. We call this parameter the saddle phase asymmetry $\Delta$. In [13] a formal proof is given of the following result for the semicycle local flow through $u=0(\bmod 1)$ :

$$
\mathcal{J}_{\text {on }}^{0}= \begin{cases}q^{-1} \operatorname{Int}\left[\operatorname{Frac}\left(q u_{\mathrm{cm}}+1 / 2\right)-q \Delta\right], & \text { if } q \text { is even, } \\ q^{-1} \operatorname{Int}\left[\operatorname{Frac}\left(q u_{\mathrm{cm}}\right)-q \Delta\right], & \text { if } q \text { is odd. }\end{cases}
$$




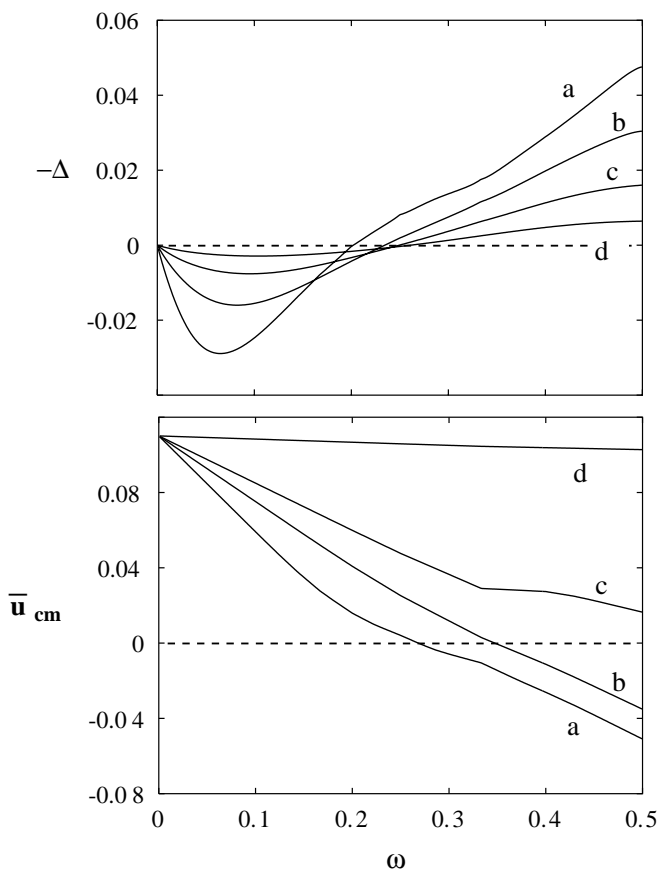

Fig. 2 - Numerical results for $-\Delta(K, \omega)$, where $\Delta$ is the saddle phase, and the mean asymmetry $\bar{u}_{\mathrm{cm}}(K, \omega)=u_{\mathrm{cm}}-1 / 2$ for $K=1,2,4,10$ labeled from a to d.

leading to the incommensurate flow:

$$
\mathcal{J}(K, \omega)=u_{\mathrm{cm}}(K, \omega)-1 / 2-\Delta(K, \omega)
$$

In physical terms, $u_{\mathrm{cm}}-1 / 2$ is simply the mean asymmetry of the modulated phase (an equilibrium $F^{*}$-average), while $\Delta$ is the position at which either the main Peierls gap starts to open during the on-relaxation, or else, the widest depression in the distribution density is originated.

Though, $u_{\mathrm{cm}}$ and $\Delta$ are determined by the competition among interactions, so that both depend on the functional details of $V$ and $W$. However, one should expect (in some sense) universality in the high-pinning limit. Indeed, as $K \rightarrow \infty$, pinning becomes overdominant, the Peierls gap opens at the position of the maximum of $V(u)$, thus $\Delta \rightarrow 0$; also, the distribution density concentrates at its minimum, so that $u_{\mathrm{cm}} \rightarrow a$. Thus one finds, independent of $\omega$ and irrespective of any particularity of $V(u)$ (other than to have a single minimum per period), the value $a-1 / 2$ for the flow of incommensurate phases in this limit. This provides a precise answer (for high pinning) to the question on the type of symmetry requirements on the modulating potential $V(u)$. On the one hand, mirror-symmetry on $V(u)$ implies $a=1 / 2$, and thus zero flow, but on the other hand, asymmetry of $V(u)$ does not guarantee nonzero flow unless $a \neq 1 / 2$, in which case transport is guaranteed for all incommensurate phases at high pinning.

Discommensuration Theory provides the finest analysis on singular features of $\mathcal{J}(K, \omega)$. Indeed, near commensurability $p / q$, the centroid $u_{\mathrm{cm}}(\omega)$ is locally given by the piecewise linear function:

$$
u_{\mathrm{cm}}(\omega)=u_{\mathrm{cm}}(p / q)-|\omega-(p / q)| q \alpha_{\text {kink }}^{ \pm}
$$




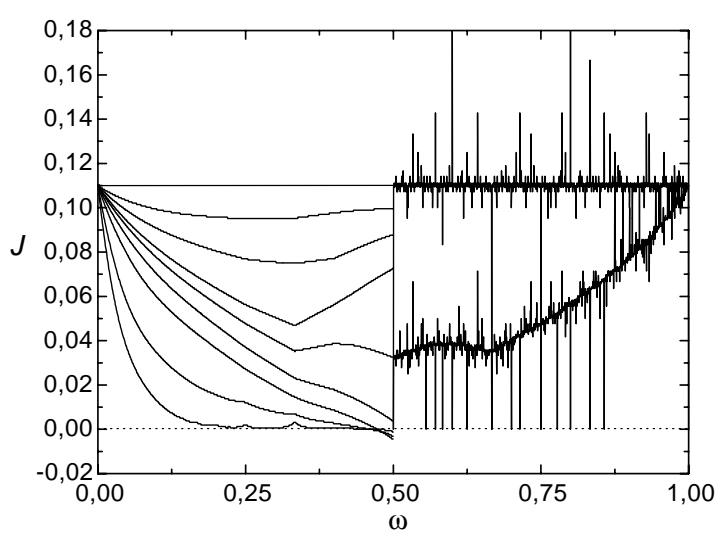

Fig. 3 - Left: incommensurate flow $\mathcal{J}(\omega)$ from eq. (8), for several values of $K=\infty, 6,5,4.5,4,3,2,1$ and 0.5. Right: commensurate flow from direct numerical integration of eqs. (1) and (2), for $K=\infty$ and $K=4$; it fits up to significant digits with the sum of (5) and (7).

where $\alpha_{\text {kink }}$ is the relative asymmetry of the discommensuration (DC) with respect to the commensurate $p / q$ configuration. The superindex \pm refers to advanced/delayed DC (respectively, $\omega>p / q$ and $\omega<p / q$ ) [14]. Formula (9) reflects the physical image of incommensurate phases as near equispaced arrays of exponentially localized phase defects (or weakly interacting DCs) over nearby commensuration. The error bound in (9) is exponentially small in the ratio DC width over DCs interspacing (see [14]). Note that when the $K$ values approach zero, the DC widens and eventually the strong overlap of neighbouring DCs shrinks the domain of (local) validity of (9). Nevertheless, from (8) and (9), one safely obtains the following: The flow surface generically presents jump discontinuities in its first partial derivative with respect to $\omega$ at all rationals, though for most commensurates the jump can be shown to be negligibly small.

In order to illustrate these complexities of $\mathcal{J}(K, \omega)$, we have numerically computed $u_{\mathrm{cm}}$ and

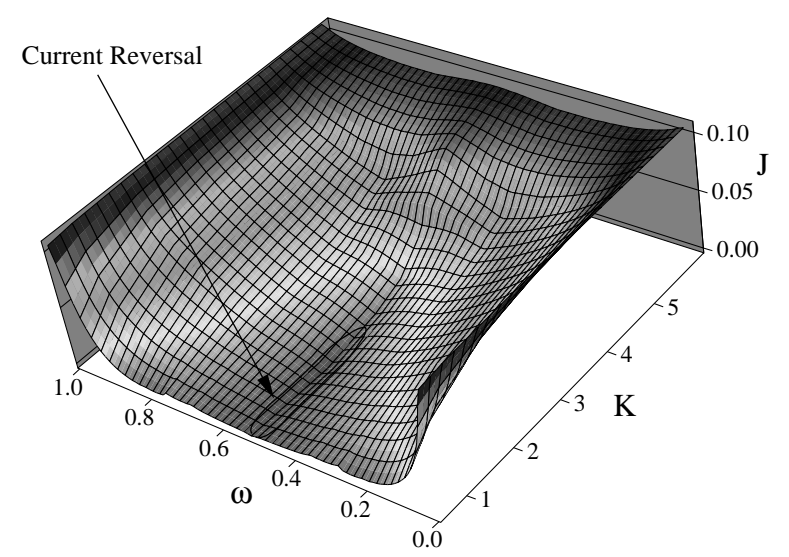

Fig. 4 - Perspective on the surface $\mathcal{J}(K, \omega)$ for $0.5 \leq K \leq 6$ and $0<\omega<1$. Its most noticeable features, namely the crests of high flow near integer commensuration and the region of current reversal at low $K$ values are due to the dominance of $u_{\mathrm{cm}}-1 / 2$ over the contribution of $\Delta$. Peaked valleys and/or crests are noticeable at some low-order rationals $\omega=0,1 / 2,1 / 3,1 / 4, \ldots$. 
$\Delta$ (which can be performed with virtually unlimited precision) for a dense grid of $\omega$-values and different values of $K$, for the specific (though arbitrary) choice of $V(u)=(2 \pi)^{-2}[\sin (2 \pi u)+$ $0.22 \sin (4 \pi u)]$, for which $a-1 / 2=0.110$, and $W(\Delta u)=1 / 2(\Delta u)^{2}$. It is not difficult to realize that $u_{\mathrm{cm}}(K, \omega)=u_{\mathrm{cm}}(K, 1+\omega)=u_{\mathrm{cm}}(K, 1-\omega)$ and $\Delta(K, \omega)=\Delta(K, 1+\omega)=\Delta(K, 1-\omega)$, so that one has only to consider values of $0 \leq \omega \leq 1 / 2$.

Our numerical results are shown in fig. 2. From there the incommensurate flow (8) is shown in the left half of fig. 3. Direct numerical integration of eqs. (1) and (2) in the right part of fig. 3 fits up to significant digits with (5) and (7), as a final test on numerical codes. In this example, for not too low values of $K$ (say, above 1 ), the term $u_{\mathrm{cm}}-1 / 2$ dominates over the $\Delta$ contribution to flow (8), so that the mean asymmetry of the modulated phase determines the most prominent features of the flow surface shown in fig. 4 . In particular, it is responsible for the current reversal near half-commensurability $(\omega=1 / 2)$ and of the crests of high flow near integer $\omega$.

The reader may take a close look at some of these singularities (notably at $\omega=0,1 / 2,1 / 3$, $1 / 4, \ldots)$ in the example shown in figs. 3 and 4 . These and other related issues will be thoroughly discussed elsewhere [13], where a rigorous exposition of the proof for (5) to (8) is given.

Summarizing, we have presented some rigorous statements on the phase transport by cyclic pumping of pinning energy for generic modulated phases of the Frenkel-Kontorova model. The type of asymmetry of the modulating field which is needed for transport has been clarified. We have also shown explicit computations of the steady-flow phase diagram whose detailed features and complex structure of singularities are explained in physically appealing terms, with bounded error, using discommensuration theory.

One can certainly find similarities between our problem and what is popularly known as ratchet effect or directed transport $[15,16]$. Here it is an example of deterministic (nonthermal) genuinely collective ratchet which has the virtue of being amenable to exact analysis. From this interesting perspective, our results could also provide a rigorous referent in connection to some important aspects currently of interest in application of ratchet transport to Condensed Matter Physics, like soliton ratchets [17] or Josephson junction ratchet devices [18].

$$
* * *
$$

This work is dedicated to Dr. Domingo GonzÁlez, of the University of Zaragoza, on the occasion of his retirement. We thank C. Baesens, J. L. García-Palacios, R. S. Mackay, J. M. SANCho and G. P. Tsironis for useful discussions on this work. Financial support is acknowledged to DGES PB98-1592 of Spain, Acción Integrada Hispano-Británica HB1999-0104, and European Network LOCNET HPRN-CT-1999-00163. LMF is grateful to the Mathematics Institute at Warwick where this work was completed.

\section{REFERENCES}

[1] Griffiths R. B., Fundamental Problems in Statistical Mechanics, Vol. VII, edited by VAN BeiJeren H. (North-Holland, Amsterdam) 1990, p. 69.

[2] Selke W., Phase Transitions and Critical Phenomena, edited by C. Domb and J. L. Lebowitz (Academic, New York) 1993.

[3] Yeomans J. M., Solid State Physics, Vol. 41, edited by Ehrenreich H. and Turnbull D. (Academic, New York) 1988.

[4] Frenkel Y. and Kontorova T., J. Phys. (USSR), 1 (1939) 137.

[5] Aubry S., in Structures et instabilités, edited by Godreche C. (Editions de Physique, Les Ulis) 1985 , p. 73. 
[6] Chaikin P. M. and Lubensky T. C., Principles of Condensed Matter Physics (Cambridge University Press) 1995.

[7] Scotт A., Nonlinear Science. Emergence and Dynamics of Coherent Structures (Oxford University Press) 1999.

[8] Floría L. M. and Mazo J. J., Adv. Phys., 45 (1996) 505.

[9] Baesens C. and Mackay R. S., Nonlinearity, 11 (1998) 949.

[10] Kuipers L. and Niederreiter H., Uniform Distribution of Sequences (John Wiley \& Sons, New York) 1974.

[11] The fractional part of a real number $x$ is defined as $\operatorname{Frac}(x)=x-\operatorname{Int}(x)$, with $\operatorname{Int}(x)$ the largest integer not greater than $x$ (integer part). If we denote by $F_{M, N}(x)$ the fraction of the indices $n(M \leq n<N)$ such that $\operatorname{Frac}\left(u_{n}\right)$ lies in $[0, x)$, then the d.f. $(\bmod 1) F(u)(0 \leq u \leq 1)$ is defined as the limit

$$
F(u)=\lim _{(N-M) \rightarrow \infty} F_{M, N}(u) .
$$

Not every conceivable sequence will give rise to a limit (10), but this limit exists for any rotationally ordered configuration $\left(u_{n}\right)$.

[12] Katok A. and Hasselblatt V., Introduction to the Modern Theory of Dynamical Systems (Cambridge University Press) 1995.

[13] Floría L. M. and BAesens C., preprint (2002).

[14] If $u_{n}^{\sigma}(\sigma= \pm)$ denotes the advanced/delayed DC configuration, $\alpha_{\text {kink }}=\sum_{n}\left(u_{\mathrm{cm}}(p / q)-\right.$ $\left.\operatorname{Frac}\left(u_{n}^{\sigma}\right)\right)$. The error in expression (9) is of the form $C \exp [-2 /(q \xi|\omega-p / q|)]$, where $\xi$ is the decay range of $p / q$ commensurate, that is, the discommensuration width.

[15] H. Linke (Editor), Appl. Phys. A, Vol. 75, special issue on Ratchets and Brownian Motors: Basics, Experiments and Applications (2002).

[16] Reinmann P., Phys. Rep., 361 (2002) 57.

[17] Costantini G. and Marchesoni F., Phys. Rev. Lett., 87 (2001) 114102; Salerno M. and Quintero N. R., Phys. Rev. E, 65 (2002) 025602; Salerno M. and Zolotaryuk Y., Phys. Rev. E, 65 (2002) 056603.

[18] Falo F., Martínez P. J., Mazo J. J. and Cilla S., Europhys. Lett., 45 (1999) 700; Trías E., Mazo J. J., Falo F. and Orlando T. P., Phys. Rev. E, 61 (2000) 2257; Waunbaugh J. F., Reichhardt C., Olson C. J., Marchesoni F. and Nori F., Phys. Rev. Lett., 83 (1999) 5106. 